\title{
Experimental Studies of Carpenter Bee (Xylocopa: Apidae) Thorax Mechanics During
}

\section{Defensive Buzzing.}

Mark Jankauski ${ }^{1}$, Cailin Casey ${ }^{1}$, Chelsea Heveran ${ }^{1}$, Kathryn Busby, and Stephen Buchmann ${ }^{2}$

Montana State University, Dept. of Mechanical \& Industrial Engineering, Bozeman, MT ${ }^{1}$

University of Arizona, Dept. of Ecology \& Evolutionary Biology, Tucson, AZ²

\begin{abstract}
Bees and other Hymenoptera utilize thorax vibration to realize an extensive range of behaviors ranging from flight to pollination. Strong indirect flight muscles contract to deform the thoracic walls and the resulting oscillation is sustained through a mechanism called stretch activation. While the mechanics of the insect thorax and muscles have been studied extensively during flight, relatively little is known about the thorax mechanics during non-flight behaviors. In this work, we investigate the thorax mechanics of the carpenter bee Xylocopa californica during defensive buzzing. During defensive buzzing, the insect folds its wings over its abdomen and rapidly fires it flight muscles, resulting in a loud audible buzz and large forces intended to deter predators. We devised a novel experiment to measure thorax oscillation and directional force production from a defensively buzzing carpenter bee. The largest peak forces were on average $175 \mathrm{mN}$ and were oriented with the insect's dorsal-ventral muscle group. Peak forces oriented with the insect's dorsal-longitudinal muscle group averaged $117 \mathrm{mN}$. Thorax velocities were about $90 \mathrm{~mm} \mathrm{~s}^{\wedge}-1 \mathrm{p}-\mathrm{p}$ and velocity amplitude was positively correlated to peak force. Thorax oscillation frequency averaged $132 \mathrm{~Hz}$ but was highly variable both within individuals and across the tested population. From our measurements, we estimated the peak mechanical power required by defensive buzzing at $8.7 \mathrm{~mW}$, which we hypothesize is greater than the power
\end{abstract}


required during flight. Overall, this study provides insight into the function and capabilities of the Hymenopteran indirect flight muscle during non-flight behaviors.

\section{Introduction}

Insects in the order Hymenoptera are globally diverse with over 150,000 extant species (Mayhew, 2007). These organisms are the wasps, bees, and ants with both solitary and social lifestyles. Most species have winged adults capable of powerful long-distance flights between their nests and widely scattered food resources. Their mid body section, the thorax, is a compact box-like region enclosed by the exoskeleton. This "armor" of hard chitin is a polymer of acetylglucosamine resistant to some chemicals and crushing mechanical forces (Elias-Neto et al., 2010). Proteins including sclerotin are interwoven and cross linked to chitin giving insect exoskeletons their unique chemical and biophysical properties (Brunet, 1980). Indeed, the exoskeletons of insects and other arthropods are likely a major factor, along with small size and fecundity, to their evolutionary success and diversity, and their preservation in the fossil record.

During flight, the flight box (pterothorax) is actively deformed by the action of internal sets of perpendicular, antagonistic fibrillar muscles known as the indirect flight muscles (IFM) (Josephson et al., 2000). These muscles are responsible for flight and other behaviors. The IFM are power-producing muscles that move the fore and hind wings indirectly by active deformation of the thoracic exoskeleton (Mountcastle \& Combes, 2013). The large fibrillar flight muscles of a bee include the dorsal-ventral muscles (DVM) and the dorsal-longitudinal muscles (DLM) (Iwamoto, 2011). The two DLM muscle sets extend nearly parallel to the long axis of the body, centrally located, and attach to the front and back surfaces of the inner thorax. 
Several Hymenoptera use their powerful IFM muscles to facilitate and regulate many essential behaviors. The most important behaviors in bees, wasps and other insects include thermoregulatory warmup for the individual (shivering) in preparation for takeoff and flight (Esch, 1960; Heath \& Adams, 1967; Heinrich \& Buchmann, 1986), airborne flight itself (Esch, 1976; Esch et al., 1975), heating of the brood nest in honey bees and bumblebees (Esch, 1960; Kleinhenz et al., 2003), vibrating soil particles during nest excavation or completion (Jane Brockmann, 1985; Spangler, 1973), forager related intranidal communication sounds in honey bees, stingless bees and bumblebees (Dornhaus \& Chittka, 2004; Nieh, 2004), buzz pollination (floral vibratile behavior) for pollen collection (Buchmann, 1983; Vallejo-Marín, 2019) and fanning to cool the nest during hot weather (Esch et al., 1991; Lindauer, 1954).

Additionally, both sexes of adult bees, ants and wasps typically respond to external threats by contracting their IFM resulting in one or more bursts of audible alarm or defensive buzzing sounds (Pritchard \& Vallejo-Marín, 2020; Vallejo-Marín \& Vallejo, 2021). Strong vibrations, often reinforced by a concurrent painful sting, may surprise and deter predators. Presumably, an alarm-buzzing bee is often released and escapes unharmed, but a literature review indicates no direct or experimental evidence of this outcome. In mutillid wasps, females stridulate (produce rasping squeaks) when caught or held - these powerful thoracic defensive buzzes/vibrations do often result in their release and escape (Schmidt \& Blum, 1977).

The physical characteristics of defensive buzzing have been investigated only in limited contexts. De Luca and Vallejo-Marin measured acoustic signatures during defensive buzzing in bumblebees and showed that buzzing characteristics varied widely across bumblebee species both in frequency and duration (de Luca et al., 2014). Vallejo-Marin and Vallejo measured thorax acceleration directly and compared the defensive buzzing frequencies and amplitudes across multiple species of flower visiting hoverflies and bees (Vallejo-Marín \& Vallejo, 2021). 
Other studies have used non-contact laser vibrometry to measure thorax oscillations during defensive buzzing and other behaviors. Hrncir et al. used laser vibrometry to record thorax oscillations in the tropical stingless bee Melipona seminigra and determined that thorax resonance was associated with flight behavior but not defensive buzzing (Hrncir et al., 2008). Pritchard and Vallejo-Marin measured thorax vibrations during defensive buzzing, buzzpollination and flight and found vibration amplitude and frequency were highest during buzz pollination, lowest during flight and in between during defensive buzzing (Pritchard \& VallejoMarín, 2020).

Although these studies have advanced our understanding of defensive buzzing, there remain open questions regarding the mechanics of this behavior. First, the magnitude of muscular forces during defensive buzzing, and in which directions those forces are generated, remain unknown. Secondly, we do not understand which features of thorax vibration, such as amplitude or frequency, are most strongly correlated to force generation. Finally, while the power requirements of flight have been the subject of considerable research, the power consumption during defensive buzzing or other non-flight behaviors has not been estimated.

The goal of the present research is to simultaneously measure thoracic vibration and directional force production during defensive buzzing in Arizona carpenter bees. We devised methods and experiments to determine what features of thoracic vibration (e.g., velocity magnitude, frequency) are most strongly correlated to increases in force production. We also identified whether the stiffness of the object to which the bee was mounted during defensive buzzing significantly influenced thorax oscillation properties or force generation. Lastly, we explored the power requirements of defensive buzzing and how these may relate to similar power requirements of buzz pollination or during unrestrained flight. While the present research 
focuses on defensive buzzing, the results provide insight into the functioning of carpenter bee flight muscles in general.

\section{Materials and Methods}

\section{2a. Specimen Collection}

Live carpenter bee adult females were collected from sites around Pima and Santa Cruz Counties (AZ) on August 20, 2020; September 27 and 29, 2020; and October 11 and 13, 2020. All Xylocopa (Xylocopoides) californica arizonensis Cresson females were obtained from within their tunnel nests inside one year old Sotol (Dasylirion wheeleri) dried inflorescence stalks (Fig. 1). In these cases, whole stalks containing nests were removed, entrances sealed with a rope knot, and brought to a lab setting where the stalks were split longitudinally with a beekeeper's hive tool. Resident bees within were then transferred immediately to $15 \mathrm{~mL}$ plastic Eppendorf tubes with added ventilation holes. Due to this collection method, all adults were most likely newly eclosed young adults. A moist piece of cardboard was added to line each Eppendorf tube to physically support the bees and for added moisture. The tubes were then refrigerated at $4^{\circ} \mathrm{C}$ for $1-6$ days. Prior to shipment, tubes were insulated with a Blue lce pack and/or Styrofoam cylinder, all of which was contained within a cardboard cylinder for shipping. Bees were shipped overnight to the site of the subsequent experiments (Montana State University, Bozeman, MT), with all shipments arriving within one week of initial field collection.

\section{2b. Experimental Design}

We developed a simple yet informative laboratory experiment to measure the directional forces and thoracic vibrations during defensive buzzing behaviors by female X.c. arizonensis. Forces were measured using a high-sensitivity six axis Nano17-E force-torque transducer (ATI 
Industrial Automation, Apex, NC, USA) capable of resolving forces down to $3.125 \mathrm{mN}$. We mounted a cylindrical post to the force transducer (Fig. 2) that bees were fixed to during experimental trials. The post was 3D printed using a FormLabs Form 2 SLA printer (Formlabs, Somerville, MA, USA) with proprietary tough resin, which has similar properties to Acrylonitrile Butadiene Styrene (ABS). The plastic post had a natural frequency of $715 \mathrm{~Hz}$. Initial testing indicated that bees produced higher-order force harmonics that extended to frequencies past the post's natural frequency. To identify if post stiffness influenced force generation or thorax vibration, we 3D printed a second post from continuous carbon fiber with $37 \%$ triangular infill using a Markforged X7 printer (Markforged, Watertown, MA, USA). The carbon fiber post increased the first resonant frequency of the system to $1400 \mathrm{~Hz}$. During experimental trials, thorax deformation velocity was measured using a non-contact laser vibrometer (Polytec, PSV400) at the thoracic scutum. The scutum had good reflectance and did not require treatment with retroflective tape or other reflectance enhancing agents.

Bees were refrigerated at $4^{\circ} \mathrm{C}$ degrees prior to experimentation. Cold anesthetized insects were mounted to a wooden toothpick by their mandibles via low temperature plastic hot glue. In some cases, the hot glue was extended to the labrum and clypeus to provide additional adhesion force so that the bee could not free itself during experimentation. The toothpick was subsequently glued to the post and trimmed to match the post's length. After mounting, the bees were left to warm up in ambient room temperature for 5-10 minutes. We then lightly pinched (with metal forceps) the bee's hind tarsus or tibia to initiate defensive buzzing and repeated when the bee quiesced. We recorded at least 2 minutes of buzzing per insect. Five subjects each were tested on the plastic and carbon fiber posts for a total of ten individuals. The bee's DVM and DLM flight muscles were approximately aligned with the force transducer's $x$ and $z$ axes respectively, and the transducer's y-axis runs medially-laterally (Fig. 2). 
The bees were free to move their body during experimentation, which posed challenges for data collection. The orientation of the head is fixed, but the bee can twist its body and repositioning its legs as it attempts to free itself from the glue. While flight muscle forces are transmitted to the post primarily through a rigid connection at the bee's mandibles, a small force component may be transmitted through the bee's legs as well. The motion of the bees' body may influence force directionality. To minimize this issue, we discarded trials where the bee moved excessively. We also discarded trials where the vibrometer signal exceeded a prescribed noise level. High vibrometer noise is associated with poor optical reflectance, which occurs when the scutum plane is not normal with respect to the measurement laser. Numeric criteria for data exclusion are described in the following section.

\section{2c. Data Analysis}

Time-series force and velocity data were acquired at a sampling rate of $10 \mathrm{kHz}$ and were post-processed in MATLAB 2020B. We applied a bandpass filter with a low frequency cut-off of $10 \mathrm{~Hz}$ and a high frequency cut-off of $2000 \mathrm{~Hz}$. Each trial was then divided into equal intervals of 1024 samples, where each interval overlaps $50 \%$ with the previous interval. We established two criteria that each interval must satisfy for inclusion in tabulated data. First, the root-mean-squared (RMS) value of the force recorded in the $\mathrm{x}$-axis must exceed $10 \mathrm{mN}$. This criterion is designed to exclude periods of quiescence. Second, the RMS value of the unfiltered velocity signal must be less than $0.5 \mathrm{~mm} / \mathrm{s}$. This criterion excludes intervals in which the optical reflectance is low, which indicates a high angle of incidence between the scutum and vibrometer laser. On average, 739 intervals per bee met the inclusion criterion $(\min =77, \max =2153$, std $=639)$. 
For each valid interval, we tabulated maximum values for forces in the $x, y$ and $z$ directions as well as thorax velocity. To determine thorax oscillation frequency, we applied a Hamming window to each velocity time series and subsequently converted the time series to the frequency domain via a Fast Fourier Transform (FFT) algorithm. The Hamming window is designed to reduce spectral leakage. We use MATLAB to identify velocity magnitude peaks that exceeded $1 \mathrm{~mm} / \mathrm{s} \mathrm{p}-\mathrm{p}$ and that were spaced at least $20 \mathrm{~Hz}$ from adjacent peaks. In most cases, the velocity magnitude occurs at some fundamental frequency and several integer harmonics thereof; we report only the dominant frequency where most of the vibrational energy occurs in the present research. A representative data set is shown in Fig. 3.

Lastly, we estimated the mechanical power required to deform the thorax during defensive buzzing. Mechanical power, defined as the rate at which work is done to a system, is a quantity central in the study of animal locomotion. Within bees, there has been little research to understand the energetic costs of non-flight behaviors, such as defensive buzzing or buzz pollination. The power $P(t)$ utilized during defensive buzzing is estimated as

$$
P(t)=F_{x}(t) V_{x}(t)
$$

where $F_{x}(t)$ is the force in the x-direction and $V_{x}(t)$ is the thorax velocity measured collinear to that force. This assumes that the force applied to the post through the bee's mandibles is equivalent to the thoracic force at the point where the velocity is measured. While simplified, the above expression provides a basic estimate that is useful in understanding how much power defensive buzzing requires relative to other vibratory behaviors, including flight and buzz pollination. We calculated peak power for each measurement interval.

\section{2d. Statistics}


To test whether measurements were affected by the post type, mixed model ANOVA was performed using bee (i.e., individual specimen ID) as a random effect and post as a fixed effect. Then, to analyze the relationship between force and velocity, a linear mixed effects model was used with velocity as a covariate and post as a fixed effect. Bee was included as a random effect to account for repeated measures. For all models, significance was set a priori to $p<$ 0.05. Responses were natural-log transformed if necessary to satisfy ANOVA assumptions of residual normality and homoscedasticity. Pearson correlations were calculated between all measures of force, velocity, frequency, and power. All statistical analyses were performed using Minitab (v.18).

\section{Results}

\section{3a. Effect of Post Material}

We found that the post material did not influence the peak force $\left(F_{x}, F_{y}, F_{z}\right)$ or velocity measurements ( $p>0.05$ for effect of post for each test). This indicates that we can average peak measurements between the carbon and plastic post samples. For both post materials, force harmonics of low magnitude extended to frequencies comparable to the system's resonant frequency. As a result, the force magnitude evaluated at frequencies near the system's resonant frequency will be amplified. The extensive higher-order harmonics indicate that force is applied to the post by the bees in short periodic pulses rather than harmonically. Nonetheless, bees did not excite the post resonance, and thus the peak forces measured experimentally reliably indicate the peak forces transmitted to the post by the bees. 


\section{3b. Thorax Velocity, Force and Power}

Mean and standard deviations for thorax oscillation frequency, peak velocity, force, and power

are shown in Tab. 1. We found an average thorax oscillation frequency of $132 \mathrm{~Hz}$ during defensive buzzing. Previous acoustic measurements identified thorax oscillation frequencies in X. californica of $107-147 \mathrm{~Hz}$ in tethered flight and $197-297 \mathrm{~Hz}$ during buzz pollination (King \& Buchmann, 1996). Across a wider range of Hymenoptera, defensive buzzing frequencies have been reported between 117 - 242 Hz (Vallejo-Marín \& Vallejo, 2021). Peak thorax velocities during defensive behaviors were about $32 \%$ lower compared to Bombus terrestris (Pritchard \& Vallejo-Marín, 2020) and 57\% lower compared to Melipona seminigra (Hrncir et al., 2008). Peak forces were largest along the $x$-axis, followed by the $z$ and $y$ axes, respectively. Peak force magnitudes in the $\mathrm{x}$-axis were on average $175 \mathrm{mN}$, considerably larger than the $6.13 \mathrm{mN}$ average carpenter bee weight $(n=9)$. Mean peak power consumption was about $8.7 \mathrm{~mW}$, corresponding to a body mass normalized peak power consumption of $13.9 \mathrm{~W} / \mathrm{kg}$.

Correlations for measured and calculated quantities are shown in Tab. 2. Buzzing frequency was not strongly correlated to peak velocity, force, or power. Peak velocity, $V_{x}$, had moderate positive correlations with $F_{x}$ and $F_{z}$, but was only weakly correlated with $F_{y} . F_{x}$ and $F_{z}$ were strongly positively correlated with one another, and modestly correlated with $F_{y}$. Power was strongly correlated with $V_{x}$ and $F_{x}$, the two quantities from which it was calculated.

\section{Discussion}


Defensive buzzing and other non-flight behaviors regulated by the IFMs are critical to the ecological role of bees, yet the mechanics of these behaviors are not well understood. We developed a novel experimental approach to simultaneously measure thorax vibration and the directional forces produced during defensive buzzing in $X$. californica. From these measurements, we established a simple estimate of the mechanical power exhausted during this behavior. Here, we discuss how our results of our experiment broadly provide insight into carpenter bee ecology.

\section{4a. Force Generation}

Our study shows that carpenter bees can produce forces nearly two orders of magnitude larger than their own weight during defensive buzzing. Given that thorax acceleration amplitudes are larger during defensive buzzing than during flight in B. terrestris (Pritchard \& Vallejo-Marín, 2020), we expect that the forces recorded during defensive buzzing in $X$. californica are significantly larger than those generated during flight. Peak forces in the x-axis averaged about $175 \mathrm{mN}$ but reached nearly $500 \mathrm{mN}$ at times. We hypothesize that the largest forces originate from DLM contraction. Previous studies have shown that the DLM/DVM muscle activation ratio is higher during buzz pollination than during flight (King et al., 1996). Contraction of the DLM muscles causes the thorax to expand along its dorsal ventral axis. We found that dorsal ventral axis expansion (as evidenced by a positive thorax velocity) is accompanied by a large force spike in the negative $x$ direction (Fig. 4), and to a lesser extent, a force spike in the negative $z$ direction. Later in the buzzing cycle, a smaller force applied in the positive $x$ direction causes the thorax to contract along the dorsal ventral axis (Fig. 4); this smaller force may be generated by the DVM muscle group. Because the DVM and DLM muscle groups form an antagonistic pair, we observed a strong correlation between forces in the $\mathrm{x}$ and $\mathrm{z}$ directions. 
Though we may expect the largest forces to occur in the negative $z$ direction if the DLM muscles primarily regulate defensive buzzing, it is possible that the flexible neck does not transmit forces as effectively in this direction. In the experiment, forces originate at the IFMs and are transmitted through the insect's neck to the post via a rigid connection at the insect's mandibles. The boundary condition imposed in the experiment does not permit the insect's head to move in the $x$-direction but does allow small motion in the $z$-direction due to rotation about the glued mandibles.

The boundary condition imposed in the experiment is similar to one a bee may experience during buzz pollination, where the bee bites a floral anther with its mandibles. This contrasts the boundary condition observed during defensive buzzing, where vibration transmission to the substrate occurs primarily through the insect's legs. Studies have suggested insect legs may behave as "shock absorbers" to reduce impact during landing (Zhang et al., 2019), which suggests that the legs would transmit force poorly. Therefore, defensive buzzing is likely to deter predators via acoustic cues first rather than vibrotactile signaling. The amount of force generated by the IFMs, while perhaps proportional to the volume of the defensive buzz, is more important to behaviors where substrate vibration transmission is higher. Of course, if the predator were to grasp or bite the buzzing bee directly, the force transmission would increase considerably - the large forces may deter the predator by vibrotactile means in this scenario.

Interestingly, we found that increases in thorax oscillation frequency did not correspond to increases in peak forces. Force production was instead correlated with increases in thorax vibration amplitude. This indicates that to impose a larger force to the post during defensive buzzing, the IFMs must pull harder against the thoracic walls and cause it to deform with a higher amplitude. This is consistent with how honey bees increase lift force during flight. Vance 
et al. showed that honey bees used a larger wing stroke amplitude when flying in a hypodense gas than they did when flying in air to account for the decrease in fluid density (Vance et al., 2014). Wingbeat frequency was not significantly affected by medium density. While they did not measure thorax deformation or velocity, it is likely that thorax compression increased to accommodate the larger stroke angle, since there is a quasi-static relationship between thorax compression and wing stroke angle in honeybees (Jankauski, 2020). Thus, oscillation amplitude appears to be a major driver in force production whereas oscillation frequency is not.

\section{4b. Frequency Modulation}

Wingbeat frequency was highly variable, both across the samples as well as within individuals. Across the tested population, the lowest mean thorax oscillation frequency was $98.1 \mathrm{~Hz}$, and the highest mean thorax oscillation frequency was $155.7 \mathrm{~Hz}$. The mean standard deviation and interquartile range for individual bees was $35.8 \mathrm{~Hz}$ and $43.9 \mathrm{~Hz}$, respectively. This suggests that individual bees could modulate their thorax oscillation frequency significantly over time and that the primary buzzing frequency may not be well described by a simple mean. To show this, we compute a short time Fourier transform for an individual buzzing over 40 seconds (Fig. 5). The thorax velocity frequency varies between $75 \mathrm{~Hz}$ and $150 \mathrm{~Hz}$, indicating that this individual varied its buzz frequency over a range of at least $75 \mathrm{~Hz}$.

The ability to modulate thorax oscillation frequency may be useful during buzz pollination. During buzz pollination, a bee bites a pollen-containing flower anther with its mandibles, disengages its wings, and rapidly actuates its IFMs to cause the anther to release pollen. The dynamic properties of the anther, and how these dynamic properties relate to the force input imparted by the buzz-pollinating bee, dictate the displacement amplitude of the vibrating anther. For example, periodic force inputs at or near the anther's resonant frequency 
will amplify the acceleration the anther experiences. In some cases, anther accelerations are positively correlated with the amount of pollen expelled during buzz pollination (King \& Buchmann, 1995). The ability to modulate thorax oscillation frequency in that range may underpin the bee's ability to exploit anther resonance and cause the anther to displace further without requiring an increase in applied force. Indeed, bumblebees have been shown to adjust their excitation frequency based on the biophysical characteristics of the flower type they are visiting during buzz pollination (Corbet \& Huang, 2014).

In addition to modulating buzz frequency, carpenter bees may extend their excitation bandwidth by delivering forces in repetitive impulsive loading rather than harmonically. Repeated impulses have frequency content that begin at the primary buzzing frequency and extend to integer harmonics thereof (Fig. 3F). In some instances, the magnitude of these harmonics may exceed that of the primary frequency, though they tend to diminish at frequencies exceeding $500 \mathrm{~Hz}$. Thus, during buzz pollination, force harmonics may excite anthers with resonant frequencies higher than the insect's primary buzzing frequency.

A large excitation bandwidth could improve the effectiveness of defensive buzzing as well. We found that velocity harmonics extended up to about $900 \mathrm{~Hz}$ (Fig. 3E), which provides a proxy for the acoustic frequencies generated during defensive buzzing. These higher frequency components may fall within the hearing frequency range of potential predators. While there is little research on the specific predators of adult X. californica, various birds prey on bumble bees (Goulson et al., 2018) and honey bees (Fry, 1983). Several birds' hearing are most sensitive between 1 - $4 \mathrm{kHz}$, though this range varies widely between species (Beason, 2004). Perhaps the low frequency acoustic emissions of flight may go undetected whereas the high frequency acoustic emission of defensive buzzing does not. Further research is needed on carpenter bee predation in general to assess this hypothesis. 


\section{4c. Power Consumption}

We developed a novel approach to calculate power consumed by the vibrating thorax and used this approach to provide one of the first estimates of energy expenditures during a non-flight behavior. Mechanical power is difficult to measure directly, and is generally estimated via (1) measuring the kinematics of the wing and subsequently calculating the inertial and aerodynamic power required to flap using a simplified aeromechanical wing model, (2) stimulating the IFMs electrically, measuring instantaneous muscle force and length, and calculating power from the IFM force-velocity relationship, or (3) measuring metabolic rates via respirometry. The first method cannot be used for non-flight behaviors since the insect's wings do not flap. The second method requires detailed knowledge of time-dependent muscle strain profiles, which are unknown in many contexts. The third method cannot account for muscle efficiency, which is believed to be low in IFMs, and thus produces poor estimates of mechanical power. The method proposed in this research, while approximate, estimates mechanical power generated by the thorax using only velocity and force measurements.

Like applied forces, instantaneous power occurred in repeated impulses rather than harmonically (Fig. 6). A buzzing cycle consisted of two power spikes, where the primary spike was generally 4 or 5 times larger in magnitude than the secondary spike. The power spikes coincide with the maximum thorax velocity and force generation (Fig. 5). The primary power spike may represent the power consumed by the DLM muscles whereas the smaller secondary spike represents power consumed by the DVM muscles. Each positive power spike is followed by a negative power dip of lower amplitude, which indicates power utilized by a passive elastic force responsible for bringing the thorax back to its undeformed equilibrium state. 
Based on our results, we hypothesize that the energetic costs of defensive buzzing are higher than the energetic costs of flight for $X$. californica. Peak power averaged about $10 \mathrm{~mW}$, but values could be significantly larger depending on the intensity of buzzing. Because power estimates are unavailable for $X$. californica in flight, we rely on data from $B$. terrestris for comparison. Josephson estimated muscle mass normalized peak power in B. terrestris at 100 W/kg through work-loop studies (Josephson et al., 2000). Assuming an insect mass of $100 \mathrm{mg}$, and a thoracic muscle mass of $30-50 \%$ of the total insect mass, this corresponds to peak powers in the range of $3-5 \mathrm{~mW}$. Though the $X$. californica used in this study had a mass of about 0.6 grams on average and thus would require increased power to fly, we expect that peak power during flight remains less than $5.0 \mathrm{~mW}$ if the insect leverages resonance to reduce energetic expenditures. Further, thorax velocities and accelerations (the latter of which is proportional to force) are much higher during defensive buzzing than during flight. Since power consumption is positively correlated to peak velocities and forces, this provides further evidence that defensive buzzing requires more power than flight. Even though defensive buzzing may be more energetically taxing relative to flight, it is perhaps a behavior performed less frequently. Insects spend a considerable time flying, and thus energetic efficiency during flight may ultimately be more critical to the insect's survival.

\section{Competing Interests}

The authors declare no competing interests.

\section{Data Availability}

Processed data is available at https://zenodo.org/record/5533016\#.YVJbp5rMKUk.

\section{Funding}


bioRxiv preprint doi: https://doi.org/10.1101/2021.09.28.462062; this version posted September 30, 2021. The copyright holder for this preprint (which was not certified by peer review) is the author/funder, who has granted bioRxiv a license to display the preprint in perpetuity. It is made available under aCC-BY-NC-ND 4.0 International license.

This research was supported the National Science Foundation under awards Nos. CMMI-

1942810 to MJ. Any opinions, findings, and conclusions or recommendations expressed in this material are those of the author(s) and do not necessarily reflect the views of the National

Science Foundation 


\section{References}

Beason, R. (2004). What Can Birds Hear? Proceedings 21st Vertebrate Pest Conference.

Brunet, P. C. J. (1980). The metabolism of the aromatic amino acids concerned in the crosslinking of insect cuticle. In Insect Biochemistry (Vol. 10, Issue 5). https://doi.org/10.1016/0020-1790(80)90082-7

Buchmann, S. L. (1983). Buzz pollination in angiosperms. In Handbook of Experimental Pollination Biology.

Corbet, S. A., \& Huang, S. Q. (2014). Buzz pollination in eight bumblebee-pollinated Pedicularis species: Does it involve vibration-induced triboelectric charging of pollen grains? Annals of Botany, 114(8). https://doi.org/10.1093/aob/mcu195

de Luca, P. A., Cox, D. A., \& Vallejo-Marín, M. (2014). Comparison of pollination and defensive buzzes in bumblebees indicates species-specific and context-dependent vibrations. Naturwissenschaften, 101(4). https://doi.org/10.1007/s00114-014-1161-7

Dornhaus, A., \& Chittka, L. (2004). Information flow and regulation of foraging activity in bumble bees (Bombus spp.). In Apidologie (Vol. 35, Issue 2). https://doi.org/10.1051/apido:2004002

Elias-Neto, M., Soares, M. P. M., Simões, Z. L. P., Hartfelder, K., \& Bitondi, M. M. G. (2010). Developmental characterization, function and regulation of a Laccase2 encoding gene in the honey bee, Apis mellifera (Hymenoptera, Apinae). Insect Biochemistry and Molecular Biology, 40(3). https://doi.org/10.1016/j.ibmb.2010.02.004

Esch, H. (1960). Über die Körpertemperaturen und den Wärmehaushalt von Apis mellifica. Zeitschrift Für Vergleichende Physiologie, 43(3). https://doi.org/10.1007/BF00298066

Esch, H. (1976). Body temperature and flight performance of honey bees in a servomechanically controlled wind tunnel. Journal of Comparative Physiology $\mathbf{A}$ A, 109(3). https://doi.org/10.1007/BF00663608

Esch, H., Goller, F., \& Heinrich, B. (1991). How do bees shiver? Naturwissenschaften, 78(7). https://doi.org/10.1007/BF01221422

Esch, H., Nachtigall, W., \& Kogge, S. N. (1975). Correlations between aerodynamic output, electrical activity in the indirect flight muscles and wing positions of bees flying in a servomechanically controlled wind tunnel. Journal of Comparative Physiology A, 100(2). https://doi.org/10.1007/BF00613966

Fry, C. H. (1983). Honeybee Predation by Bee-Eaters, with Economic Considerations. Bee World, 64(2). https://doi.org/10.1080/0005772x.1983.11097918

Goulson, D., O'connor, S., \& Park, K. J. (2018). The impacts of predators and parasites on wild bumblebee colonies. Ecological Entomology, 43(2). https://doi.org/10.1111/een.12482

Heath, J. E., \& Adams, P. A. (1967). Regulation of heat production by large moths. Journal of Experimental Biology, 47(1). https://doi.org/10.1242/jeb.47.1.21

Heinrich, B., \& Buchmann, S. L. (1986). Thermoregulatory physiology of the carpenter bee, Xylocopa varipuncta. Journal of Comparative Physiology B, 156(4). https://doi.org/10.1007/BF00691042

Hrncir, M., Gravel, A. I., Schorkopf, D. L. P., Schmidt, V. M., Zucchi, R., \& Barth, F. G. (2008). Thoracic vibrations in stingless bees (Melipona seminigra): Resonances of the thorax 
influence vibrations associated with flight but not those associated with sound production. Journal of Experimental Biology, 211(5). https://doi.org/10.1242/jeb.013920

Iwamoto, H. (2011). Structure, function and evolution of insect flight muscle. In Biophysics (Vol. 7). https://doi.org/10.2142/biophysics.7.21

Jane Brockmann, H. (1985). Tool Use in Digger Wasps (Hymenoptera: Sphecinae). Psyche (New York), 92(2-3). https://doi.org/10.1155/1985/73184

Jankauski, M. A. (2020). Measuring the frequency response of the honeybee thorax. Bioinspiration and Biomimetics, 15(4). https://doi.org/10.1088/1748-3190/ab835b

Josephson, R. K., Malamud, J. G., \& Stokes, D. R. (2000). Asynchronous muscle: A primer. In Journal of Experimental Biology (Vol. 203, Issue 18). https://doi.org/10.1242/jeb.203.18.2713

King, M. J., \& Buchmann, S. L. (1995). Bumble bee-initiated vibration release mechanism of Rhododendron pollen. American Journal of Botany, 82(11). https://doi.org/10.2307/2445867

King, M. J., \& Buchmann, S. L. (1996). Sonication Dispensing of Pollen from Solanum laciniatum Flowers. Functional Ecology, 10(4). https://doi.org/10.2307/2389937

King, M. J., Buchmann, S. L., \& Spangler, H. (1996). Activity of asynchronous flight muscle from two bee families during sonication (buzzing). Journal of Experimental Biology, 199(10), 2317-2321. https://doi.org/10.1242/jeb.199.10.2317

Kleinhenz, M., Bujok, B., Fuchs, S., \& Tautz, J. (2003). Hot bees in empty broodnest cells: Heating from within. Journal of Experimental Biology, 206(23). https://doi.org/10.1242/jeb.00680

Lindauer, M. (1954). Temperaturregulierung und Wasserhaushalt im Bienenstaat. Zeitschrift Für Vergleichende Physiologie, 36(4). https://doi.org/10.1007/BF00345028

Mayhew, P. J. (2007). Why are there so many insect species? Perspectives from fossils and phylogenies. In Biological Reviews (Vol. 82, Issue 3). https://doi.org/10.1111/j.1469185X.2007.00018.x

Mountcastle, A. M., \& Combes, S. A. (2013). Wing flexibility enhances load-lifting capacity in bumblebees. Proceedings of the Royal Society B: Biological Sciences, 280(1759). https://doi.org/10.1098/rspb.2013.0531

Nieh, J. C. (2004). Recruitment communication in stingless bees (Hymenoptera, Apidae, Meliponini). In Apidologie (Vol. 35, Issue 2). https://doi.org/10.1051/apido:2004007

Pritchard, D. J., \& Vallejo-Marín, M. (2020). Floral vibrations by buzz-pollinating bees achieve higher frequency, velocity and acceleration than flight and defence vibrations. Journal of Experimental Biology, 223(11). https://doi.org/10.1242/jeb.220541

Schmidt, J. O., \& Blum, M. S. (1977). Adaptations and responses of Dasymutilla occidentalis (Hymenoptera: mutillidae) to predators. Entomologia Experimentalis et Applicata, 21(2), 99-111. https://doi.org/10.1111/j.1570-7458.1977.tb02663.x

Spangler, H. G. (1973). Vibration Aids Soil Manipulation in Hymenoptera. Source Journal of the Kansas Entomological Society, 46(2).

Vallejo-Marín, M. (2019). Buzz pollination: studying bee vibrations on flowers. In New Phytologist (Vol. 224, Issue 3). https://doi.org/10.1111/nph.15666

Vallejo-Marín, M., \& Vallejo, G. C. (2021). Comparison of defence buzzes in hoverflies and buzz-pollinating bees. Journal of Zoology, 313(4). https://doi.org/10.1111/jzo.12857 
Vance, J. T., Altshuler, D. L., Dickson, W. B., Dickinson, M. H., \& Roberts, S. P. (2014). Hovering flight in the honeybee apis mellifera: Kinematic mechanisms for varying aerodynamic forces. Physiological and Biochemical Zoology, 87(6). https://doi.org/10.1086/678955

Zhang, K., Chermprayong, P., Tzoumanikas, D., Li, W., Grimm, M., Smentoch, M., Leutenegger, S., \& Kovac, M. (2019). Bioinspired design of a landing system with soft shock absorbers for autonomous aerial robots. Journal of Field Robotics, 36(1). https://doi.org/10.1002/rob.21840 


\section{Figures/Tables}

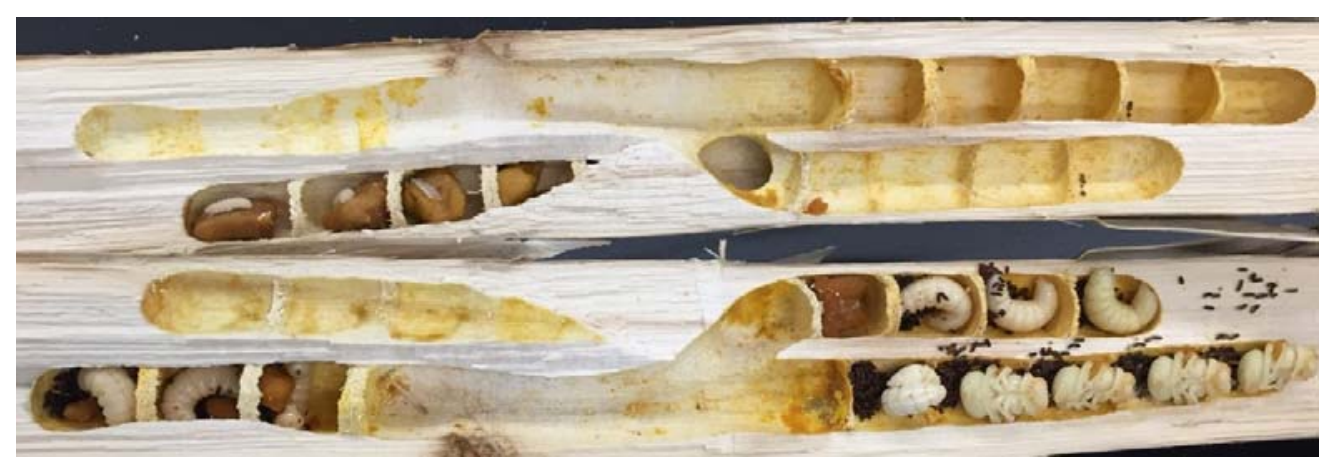

Fig. 1: Dried Sotol stalks where carpenter bees were collected. Note the presence of larvae and pupae.
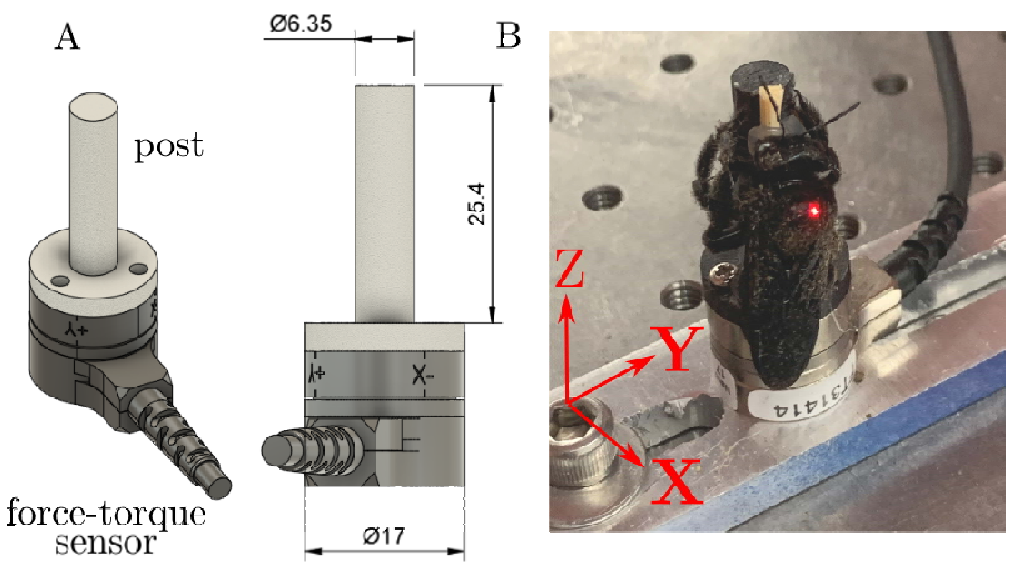

Fig. 2: Experimental set-up. (A) CAD model. Dimensions are in millimeters. (B) Carpenter bee mounted to a carbon fiber post. Note the vibrometer laser spot on the dorsal surface of the bee thorax. The cartesian basis shown in the lower left corner defines force directions. The experimental $x$ and $z$ axes most closely align with the insect's DVM and DLM muscle groups, respectively. 


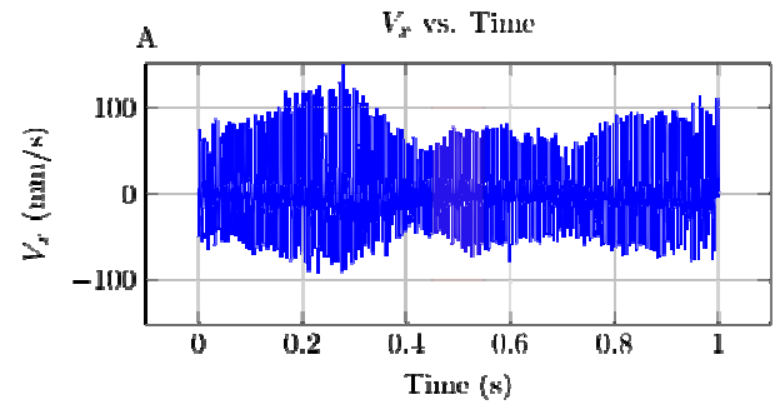

c.

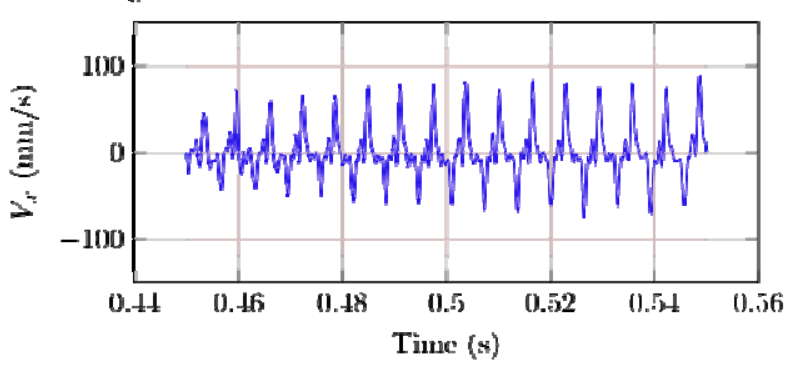

E

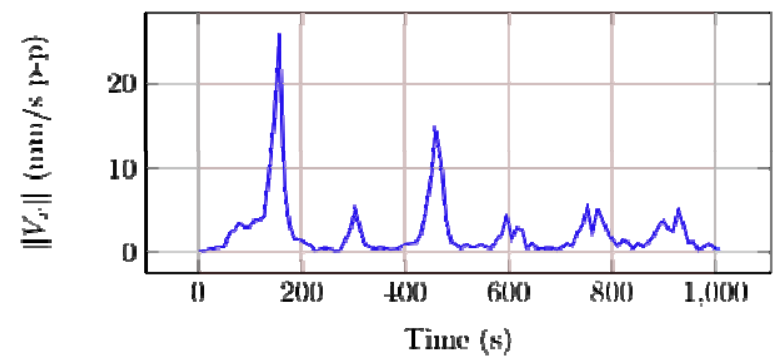

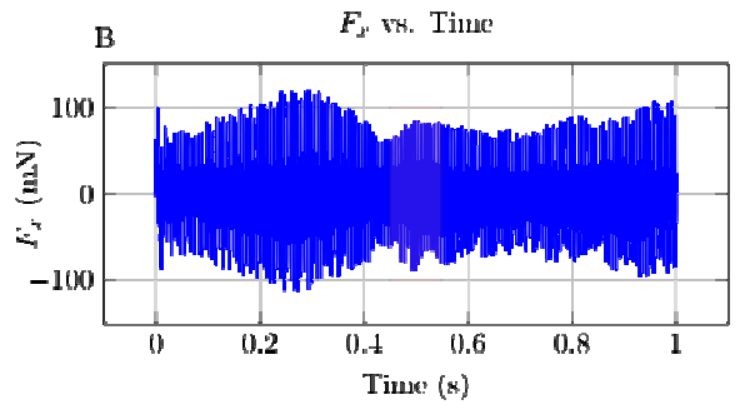

$F_{x}$ vs. Time" (interal)

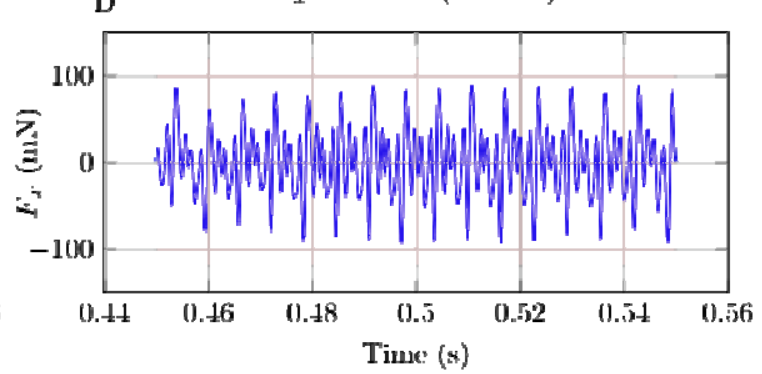

F $\quad\left\|F_{x}\right\|$ w. Frequency (interval)

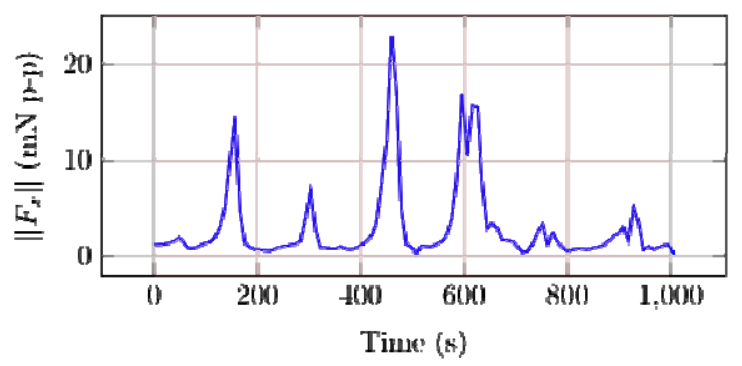

Fig. 3: Representative data showing thorax $(A, C, E)$ velocity and $(B, D, F)$ force in the $x$-axis (A-B) Time series data over one second of continuous buzzing. Note that thorax force increases proportionally with velocity. (C-D) Interval data from which oscillation frequency and peak force, velocity and power quantities are calculated from. (E-F) FFT magnitude of interval data. Thorax oscillation frequency is estimated from each velocity interval FFT. Both velocity and force exhibit significant frequency harmonics superimposed on the fundamental oscillation frequency. 


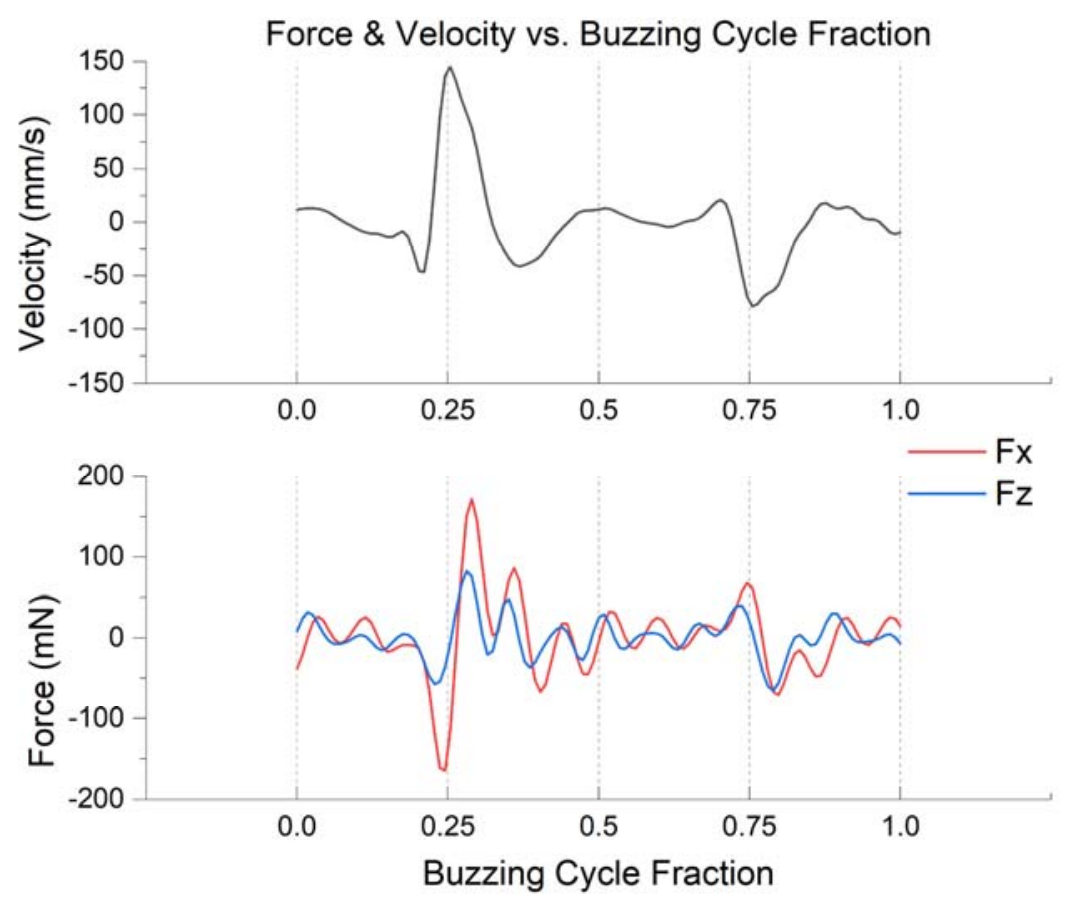

Fig. 4: Representative force and velocity traces over a single buzzing cycle. We hypothesize that the DLM muscle group contracts at $25 \%$ of the buzzing cycle, causing the thorax to expand about its dorsal-ventral axis, and the DVM muscle group contracts around $75 \%$ of the buzzing cycle, causing the thorax to expand about its longitudinal axis. The free vibration of the post can be observed in the force trace following the initial force impulse.

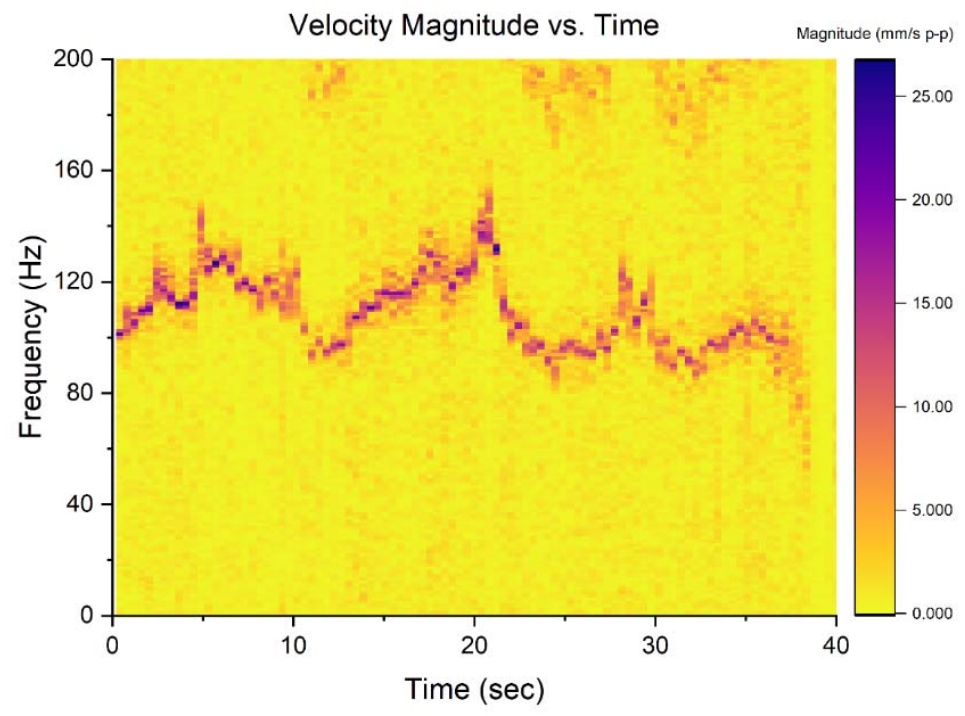


Fig. 5: Short time Fourier transform (window length $=8192$ samples, overlap $=4096$ samples, Hanning window) shows an individual bee modulating its thorax oscillation frequency between approximately $75 \mathrm{~Hz}$ and $150 \mathrm{~Hz}$.

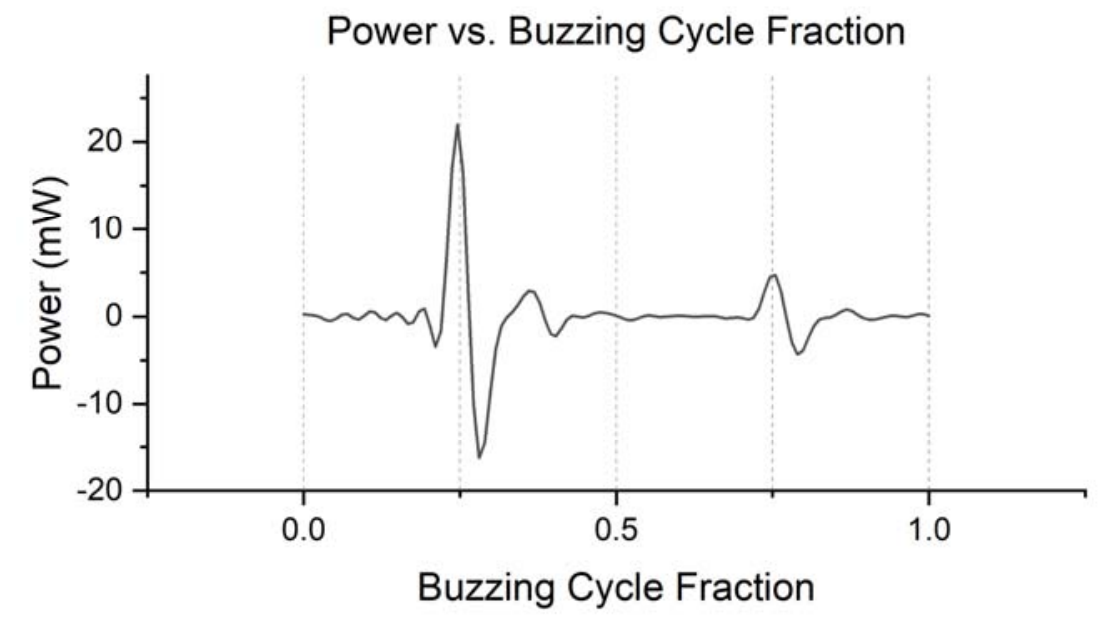

Fig. 6: Representative power trace over a single buzzing cycle. We hypothesize that the primary power spike at $25 \%$ of the buzzing cycle results from DLM contraction, and that the secondary spike at $75 \%$ of the buzzing cycle results from DVM contraction.

Table 1. Tabulated values for thorax oscillation and force measurements and power calculations. All quantities indicate peak values except thorax frequency. $P$ values are from the mixed-model ANOVA, with random effect of specimen and fixed effect of post. NS = not significant; $p>0.05$.

\begin{tabular}{|c|l|l|l|l|}
\hline Quantity & Mean & $\begin{array}{l}\text { Standard } \\
\text { Deviation }\end{array}$ & Q1 & Q3 \\
\hline$(\mathbf{m N})$ & 175.6 & 68.2 & 129.6 & 212.0 \\
Specimen: $p<0.05$ & & & & \\
\hline
\end{tabular}




\begin{tabular}{|l|l|l|l|l|}
\hline Post: NS & & & & \\
\hline $\begin{array}{l}\boldsymbol{F}_{\boldsymbol{y}}(\mathrm{mN}) \\
\text { Specimen: } \mathrm{p}<0.05 \\
\text { Post: NS }\end{array}$ & 80.5 & 35.2 & 55.0 & 101.9 \\
\hline $\begin{array}{l}\boldsymbol{F}_{\mathbf{z}}(\mathrm{mN}) \\
\text { Specimen: } \mathrm{p}<0.05 \\
\text { Post: NS }\end{array}$ & 117.3 & 49.2 & 80.6 & 145.1 \\
\hline $\begin{array}{l}\text { Frequency (Hz) } \\
\text { Specimen: } \mathrm{p}<0.05 \\
\text { Post: NS }\end{array}$ & 132.0 & 38.8 & 107.4 & 156.3 \\
\hline $\begin{array}{l}\text { Velocity (mm s^-1) } \\
\text { Specimen: } \mathrm{p}<0.05 \\
\text { Post: NS }\end{array}$ & 89.2 & 33.2 & 65.0 & 106.9 \\
\hline $\begin{array}{l}\text { Power (mW) } \\
\text { Specimen: } \mathrm{p}<0.05 \\
\text { Post: NS }\end{array}$ & 8.7 & 7.2 & 4.3 & 10.5 \\
\hline
\end{tabular}

Table 2. Correlation table showing relationships between measurements. Reported values are

Pearson correlation coefficients. All quantities indicate peak values except frequency.

\begin{tabular}{|l|l|l|l|l|l|l|}
\hline & Power $(\mathbf{m W})$ & $\boldsymbol{F}_{\boldsymbol{z}}(\mathrm{mN})$ & $\boldsymbol{F}_{\boldsymbol{y}}(\mathbf{m N})$ & $\boldsymbol{F}_{\boldsymbol{x}}(\mathrm{mN})$ & $\boldsymbol{V}_{\boldsymbol{x}}(\mathbf{m m} / \mathbf{s})$ & $\mathbf{F}(\mathbf{H z})$ \\
\hline $\mathbf{f}(\mathbf{H z})$ & 0.02 & 0.07 & 0.08 & 0.12 & 0.21 & - \\
\hline $\boldsymbol{V}_{\boldsymbol{x}}(\mathrm{mm} / \mathbf{s})$ & 0.65 & 0.36 & 0.16 & 0.40 & - & \\
\hline $\boldsymbol{F}_{\boldsymbol{x}}(\mathrm{mN})$ & 0.80 & 0.85 & 0.37 & - & & \\
\hline $\boldsymbol{F}_{\boldsymbol{y}}(\mathrm{mN})$ & 0.19 & 0.39 & - & & & \\
\hline $\boldsymbol{F}_{\boldsymbol{z}}(\mathrm{mN})$ & 0.65 & - & & & & \\
\hline Power $(\mathbf{m W})$ & - & & & & & \\
\hline
\end{tabular}


bioRxiv preprint doi: https://doi.org/10.1101/2021.09.28.462062; this version posted September 30, 2021. The copyright holder for this preprint (which was not certified by peer review) is the author/funder, who has granted bioRxiv a license to display the preprint in perpetuity. It is made available under aCC-BY-NC-ND 4.0 International license. 